\title{
Are the Institute of Medicine weight gain targets applicable in women with gestational diabetes mellitus?
}

\author{
Tang Wong ${ }^{1,2,3}$ • Robyn A. Barnes ${ }^{1,4}$ - Glynis P. Ross ${ }^{1,3}$ - Ngai W. Cheung ${ }^{3,5}$ • \\ Jeff R. Flack ${ }^{1,2,6}$
}

Received: 22 August 2016 / Accepted: 16 November 2016/Published online: 9 December 2016

(C) Springer-Verlag Berlin Heidelberg 2016

\begin{abstract}
Aims/hypothesis Our aim was to study the relationship between excessive gestational weight gain (GWG) according to Institute of Medicine (IOM) targets and perinatal outcomes, and examine whether modifying targets may improve outcomes in women with gestational diabetes mellitus (GDM). Methods This was a retrospective cohort study of all GDM pregnancies from 1992 to 2013. ORs were calculated for associations between excessive GWG (EGWG) using IOM targets and adverse pregnancy outcomes. ORs were then adjusted for maternal age, gestational age at diagnosis, prepregnancy BMI, gravidity, parity, ethnicity, antenatal fasting blood glucose level (BGL), $2 \mathrm{~h}$ BGL and $\mathrm{HbA}_{1 \mathrm{c}}$. BMI was categorised into underweight $\left(<18.5 \mathrm{~kg} / \mathrm{m}^{2}\right)$, healthy weight $\left(18.5-24.9 \mathrm{~kg} / \mathrm{m}^{2}\right)$, overweight $\left(25-29.9 \mathrm{~kg} / \mathrm{m}^{2}\right)$ and obese $\left(\geq 30 \mathrm{~kg} / \mathrm{m}^{2}\right)$. Large for gestational age (LGA) was defined as birthweight above the 90th
\end{abstract}

Electronic supplementary material The online version of this article (doi:10.1007/s00125-016-4173-3) contains peer-reviewed but unedited supplementary material, which is available to authorised users.

Tang Wong

drwongt@gmail.com

1 Diabetes Centre, Bankstown-Lidcombe Hospital, 68 Eldridge Rd, Bankstown, NSW 2200, Australia

2 Faculty of Medicine, University of New South Wales, Sydney, NSW, Australia

3 Faculty of Medicine, University of Sydney, Sydney, NSW, Australia

4 School of Health Sciences, Faculty of Health and Medicine, The University of Newcastle, Callaghan, NSW, Australia

5 Department of Diabetes and Endocrinology, Westmead Hospital, Westmead, NSW, Australia

6 School of Medicine, Western Sydney University, Sydney, NSW, Australia percentile, small for gestational age (SGA) was birthweight below the 10th percentile, macrosomia was birthweight $>4000 \mathrm{~g}$, and preterm delivery was delivery prior to 37 weeks' gestation. Modified GWG targets were derived by: (1) subtracting $2 \mathrm{~kg}$ from the upper IOM target only; (2) subtracting $2 \mathrm{~kg}$ from both upper and lower targets; (3) using the interquartile range of maternal GWG of women with infants who were appropriate for gestational age per BMI category; and (4) restricting GWG to $0-4 \mathrm{~kg}$ in women with $\mathrm{BMI} \geq 35 \mathrm{~kg} / \mathrm{m}^{2}$. Results Among 3095 GDM pregnancies, only $31.7 \%$ had GWG within IOM guidelines. Adjusted ORs for women who exceeded GWG were Caesarean section (1.5; 95\% CI 1.2, 1.9), LGA (1.8; $95 \%$ CI $1.4,2.4)$ and macrosomia $(2.3 ; 95 \%$ CI $1.6,3.3)$; there was a lower risk of SGA (adjusted OR 0.5; 95\% CI 0.3, 0.7). Conclusions/interpretation EGWG according to IOM targets was associated with Caesarean section, LGA and macrosomia. Modification of IOM criteria, including more restrictive targets, did not improve perinatal outcomes.

Keywords Gestational diabetes mellitus · Gestational weight gain $\cdot$ Institute of Medicine $\cdot$ Large for gestational age Perinatal outcomes $\cdot$ Pregnancy
Abbreviations
AGA Appropriate for gestational age
BGL Blood glucose level
EGWG Excessive gestational weight gain
GDM Gestational diabetes mellitus
GWG Gestational weight gain
IOM Institute of Medicine
LGA Large for gestational age
SGA Small for gestational age 


\section{Introduction}

The rising prevalence of obesity in childbearing age women has culminated in increasing rates of gestational diabetes mellitus (GDM) [1]. GDM in New South Wales (Australia) increased from 5.4\% in 2009 to $7.4 \%$ in 2012 [2]. Although obesity is only potentially modifiable before pregnancy, two other key maternal metabolic factors that contribute to a greater risk of adverse perinatal outcomes are modifiable during pregnancy, namely hyperglycaemia and gestational weight gain (GWG).

Appropriate GWG has been a topical issue in women of childbearing age. The recommendations for GWG during a singleton pregnancy have shifted from a single target [3] to stratified ranges based on prepregnancy BMI, first published by the Institute of Medicine (IOM) in 1990 [4] and revised in 2009 [5]. Excessive GWG (EGWG) has been demonstrated to be associated with an increased risk of pre-eclampsia, Caesarean section and large for gestational age (LGA) infants, and is linked to greater adiposity in childhood in pregnancies not complicated by GDM [6, 7]. The GWG target ranges are intended for women of all ethnicities and statures [5].

Critics have suggested that the IOM GWG targets may be overly generous, particularly in class II and III obesity, where a GWG target of $0-4 \mathrm{~kg}$ has been recommended [8]. Opposition to the IOM guidelines partly stems from the concern that there is an overemphasis on inadequate GWG and its association with low birthweight, and the lack of consideration for the long-term effects of EGWG particularly on maternal morbidity. Furthermore, there is concern that recommending a single target for all obese women may not recognise the continuum of risk that exists beyond a BMI of $30 \mathrm{~kg} / \mathrm{m}^{2}$, and stratification of risk may need to be extended for different obesity classes [9]. A more restrictive GWG target may be especially important for women with a higher BMI, as EGWG has also been associated with postpartum maternal weight retention and the subsequent risk of becoming overweight [10].

The IOM GWG guidelines, although not specific for GDM pregnancies, are frequently applied, as no GDM-specific GWG guidelines currently exist. Studies in women with GDM demonstrated that the OR of LGA was 1.72-2.01 for women who gained weight above the IOM guidelines [10, 11]. In addition, we have shown that prepregnancy BMI and maternal GWG are significant predictors of LGA [12]. It is unknown whether IOM recommendations can be applied to women who have a higher background risk of adverse outcomes, or whether adjusting these guidelines for GDM women could improve outcomes. It may be postulated that women with GDM require more stringent GWG targets due to the association and potentially additive effects of EGWG and hyperglycaemia on adverse pregnancy outcomes. We have therefore sought to determine whether weight gain targets more stringent than those recommended by IOM might be associated with a reduction in the risk of adverse outcomes such as Caesarean section, preterm delivery, LGA, shoulder dystocia, neonatal hypoglycaemia, neonatal jaundice and postpartum maternal dysglycaemia.

\section{Methods}

This was a retrospective cohort study of women with a singleton GDM pregnancy managed between 1992 and 2013 at Bankstown-Lidcombe Hospital, Sydney, NSW, Australia, supervised by the same endocrinologist and employing the same diagnostic criteria and treatment targets. The population was a multiethnic cohort of women with a high background rate of GDM and type 2 diabetes mellitus [13, 14].

Following GDM diagnosis, women received education from a diabetes educator and a dietitian in either an individual or a group setting, followed by at least one additional individual dietitian appointment. GWG targets were discussed as part of the dietary assessment and at subsequent health professional visits. Women were weighed at each visit. Self-monitoring of blood glucose level (BGL) four times daily was undertaken, and women were reviewed in a multidisciplinary clinic (including by an endocrinologist) every $1-2$ weeks. If glycaemic targets were not achieved (fasting $\mathrm{BGL}$ $<5.5 \mathrm{mmol} / \mathrm{l} ; 1 \mathrm{~h} \mathrm{BGL}<7.4 \mathrm{mmol} / \mathrm{l} ; 2 \mathrm{~h} \mathrm{BGL}<7.0 \mathrm{mmol} / \mathrm{l}$ ), insulin was commenced using intermediate-acting human NPH insulin, insulin aspart (Novorapid, Baulkham Hills, NSW, Australia, manufactured by Novo Nordisk Pharmaceuticals) or insulin lispro (Humalog, West Ryde, NSW, Australia, manufactured by Eli Lilly). Oral hypoglycaemic agents were not used.

GDM was defined according to the 1998 Australasian Diabetes in Pregnancy Society criteria [15], which had been in use since 1991 [16]. These were fasting BGL $\geq 5.5 \mathrm{mmol} / \mathrm{l}$ and/or $2 \mathrm{~h} \mathrm{BGL} \geq 8.0 \mathrm{mmol} / \mathrm{l}$ after a $75 \mathrm{~g}$ OGTT [15]. We analysed a database extract of the following data collected prospectively during routine healthcare: age, gravidity, parity, ethnicity, gestational age at diagnosis, prepregnancy BMI (based on self-reported weight recorded at first clinic visit), OGTT results, $\mathrm{HbA}_{1 \mathrm{c}}$ at GDM diagnosis, last clinic weight recorded within 4 weeks of delivery, treatment modality, mode of delivery, infant birthweight and postpartum OGTT results (from those who attended, 59.4\%). Exclusion criteria were patients who delivered before 36 weeks' gestation; no recorded data within 4 weeks of delivery. GWG was calculated as self-reported prepregnancy weight subtracted from the last recorded weight within 4 weeks of delivery. We assessed GWG relative to IOM recommendations as less than IOM, consistent with IOM, or in excess of IOM targets across four BMI categories: underweight (BMI $<18.5 \mathrm{~kg} / \mathrm{m}^{2}$ ); healthy weight (BMI $\geq 18.5$ to $\leq 24.9 \mathrm{~kg} / \mathrm{m}^{2}$ ); overweight (BMI $\geq 25$ 
to $\leq 29.9 \mathrm{~kg} / \mathrm{m}^{2}$ ); obese (BMI $\geq 30 \mathrm{~kg} / \mathrm{m}^{2}$ ). These guidelines recommend GWG of 12.5-18.0 kg in underweight women, $11.5-16.0 \mathrm{~kg}$ in healthy weight women, $7.0-11.5 \mathrm{~kg}$ in overweight women and 5.0-9.0 kg in obese women [5].

Birthweight percentiles were ethnicity specific and derived from an online customised weight centile calculator (Bulk Centile Calculator, version 6.7.3_13, www.gestation. net/index.htm, accessed February 2014). Birthweight was categorised into three groups: small for gestational age (SGA), defined as birthweight below the 10th percentile; appropriate for gestational age (AGA), defined as birthweight between the 10th and 90th percentiles; and LGA, defined as birthweight above the 90th percentile. Macrosomia was defined as birthweight $>4000 \mathrm{~g}$. $\mathrm{HbA}_{1 \mathrm{c}}$ data (mean $\pm \mathrm{SD}$ ) were converted from per cent to $\mathrm{mmol} / \mathrm{mol}$ using the public domain National Glycohemoglobin Standardization Program website converter (www.ngsp. org/convert1.asp, accessed 16 July 2015). Preterm delivery was defined as delivery prior to 37 weeks' gestation.

To determine whether IOM guidelines affected outcomes in our GDM cohort, ORs were calculated for EGWG on the risk of insulin therapy, preterm delivery, Caesarean section, LGA, macrosomia, SGA, neonatal hypoglycaemia, neonatal jaundice and postpartum maternal dysglycaemia. The reference group was women who had appropriate GWG as defined by IOM guidelines for prepregnancy BMI. ORs were also adjusted for potential biologically plausible confounders including maternal age, gestational age at diagnosis, gravidity, parity, ethnicity, prepregnancy BMI, fasting BGL, $2 \mathrm{~h}$ BGL and $\mathrm{HbA}_{1 \mathrm{c}}$ for each adverse pregnancy outcome.

To determine whether adjustments to current IOM (2009) targets (including smaller recommended GWG ranges) would have been associated with improved outcomes in women in our cohort who achieved IOM guidelines, we undertook several analyses using different GWG targets within each BMIspecific GWG range, derived by: (1) subtracting $2 \mathrm{~kg}$ from the upper IOM target only; (2) subtracting $2 \mathrm{~kg}$ from both upper and lower targets; (3) using the interquartile range (25th-75th percentile) of maternal GWG of women with AGA infants and applying that as the 'target' in each BMI category; and (4) restricting the GWG target to $0-4 \mathrm{~kg}$ in class II and III obesity (BMI $\geq 35 \mathrm{~kg} / \mathrm{m}^{2}$ ), as recommended by Kiel et al [8].

Our study was approved by the Human Research Ethics Committee of the South Western Sydney Local Health District.

\section{Statistical analysis}

Data are expressed as mean $\pm \mathrm{SD}$ or median (range). Unpaired $t$ tests or ANOVA with Bonferroni adjustment were undertaken for multiple comparisons of continuous variables; $\chi^{2}$ analyses (or Fisher's exact test if any cell was less than 5) were used to assess statistical significance in continuous and categorical variables, respectively. A $p$ value of $<0.05$ was considered statistically significant. To assess the precision of each OR, 95\% CIs were computed. Multiple logistic regression models were used to obtain adjusted ORs. All analyses were performed using IBM SPSS Statistics software, version 23.0 (IBM, Armonk, NY, USA).

\section{Results}

\section{Variability of weight gain in women with GDM}

There was a total of 3095 GDM pregnancies in 2638 women. GWG and BMI data were available for 3031 pregnancies in 2581 women. The mean age was $32.1 \pm 5.3$ years and mean gestational age at GDM diagnosis was $27.0 \pm 5.9$ weeks. East and South-East Asian (34.4\%) was the most common ethnicity, followed by Middle Eastern (27.0\%), European (22.4\%) and South Asian (11.0\%). Other ethnicities (African subcontinent and Pacific Islanders) constituted 5.3\% of the total population studied. The GDM population contained more women with a non-European ethnic background than the general obstetric patient cohort.

Across all prepregnancy BMI categories, mean GWG was $12.3 \pm 6.1 \mathrm{~kg}$. The mean prepregnancy BMI was $26.3 \pm 6.4 \mathrm{~kg} / \mathrm{m}^{2}$. GWG decreased with increasing prepregnancy $\mathrm{BMI}$; hence, there was a negative correlation between GWG and prepregnancy BMI (Pearson's correlation $-0.15, p<0.001)$.

\section{EGWG in women with GDM according to IOM weight gain targets}

GWG was categorised according to IOM GWG targets for the entire pregnancy. In only $31.7 \%$ of all pregnancies (962 of 3031) did women gain according to IOM recommendations. The largest proportion of pregnancies $(38.0 \% ; 1151$ of 3031) achieved a GWG in excess of IOM recommendations. A greater proportion of women with a healthy range BMI were able to achieve GWG according to IOM targets (35.9\%; 503 of 1401 women) compared with those who were overweight $(31.4 \% ; 257$ of 818 women; $p<0.05)$ and obese $(21.6 \% ; 150$ of 696 women; $p<0.001)$. A total of $58.5 \%$ of pregnancies (407 of 696) in women with prepregnancy BMI in the obese range had EGWG.

Rates of EGWG differed by ethnicity. Compared with women of European descent, those of East and South-East Asian and South Asian descent were less likely to exceed IOM GWG guidelines: $18.4 \%$ vs $46.3 \%(p<0.001)$ and $10.5 \%$ vs $46.3 \%(p<0.01)$, respectively. By contrast, women of Middle Eastern descent were more likely to exceed IOM GWG guidelines: $52.7 \%$ vs $46.3 \%$, respectively $(p<0.01)$. 
A large proportion of women with an elevated BMI had already exceeded their IOM total GWG target by the time of GDM diagnosis. In women who were overweight, $36.5 \%$ had already gained in excess of the $11.5 \mathrm{~kg}$ upper limit suggested for the entire duration of pregnancy. In obese women, $47.8 \%$ had exceeded the $9.0 \mathrm{~kg}$ upper limit of GWG suggested by IOM at GDM diagnosis.

\section{Baseline antenatal characteristics}

On univariate analysis, younger age, non-European descent, higher gravidity, parity, prepregnancy BMI, fasting BGL, $\mathrm{HbA}_{1 \mathrm{c}}$ and insulin therapy during pregnancy were associated with EGWG (all covariates, $p<0.01$ ) (Table 1). On multivariable analysis, younger age, higher gravidity, elevated prepregnancy BMI and elevated fasting BGL were independent predictors of EGWG.

\section{Effects of EGWG on pregnancy outcomes}

In women exceeding GWG targets, compared with those with appropriate GWG, the unadjusted OR of requiring insulin therapy was 1.7 (95\% CI 1.5, 2.0). However, after adjustment for age, ethnicity, gestational age at diagnosis, gravidity, parity, prepregnancy BMI, fasting and $2 \mathrm{~h} \mathrm{BGL}$ and $\mathrm{HbA}_{1 \mathrm{c}}$, using a multivariable logistic regression model, EGWG was no longer a significant independent predictor of need for insulin therapy (Table 2).
GWG above recommended IOM targets also increased the OR for Caesarean section (OR 1.4; 95\% CI 1.2, 1.7). When adjusted for the same covariates as above, the OR for Caesarean section was 1.5 (95\% CI 1.2, 1.9). The OR for shoulder dystocia was 3.4 (95\% CI 1.2, 10.0) and 3.0 (95\% CI, 0.7, 12.6) after adjustment.

The OR for LGA offspring in women exceeding IOM targets was 2.0 (95\% CI 1.6, 2.5) compared with those with appropriate GWG. The mean birthweight was higher in women who exceeded IOM GWG (3494 g vs $3291 \mathrm{~g}, p<0.001$ ). The adjusted OR for LGA for women exceeding IOM GWG targets was 1.8 (95\% CI 1.4, 2.4). Among women represented by the four largest ethnicities, those of South Asian ethnicity had the highest association between LGA and EGWG compared with those with appropriate GWG (adjusted OR 2.5; 95\% CI 1.1, 5.9), followed by women of European descent (adjusted OR 2.2; $95 \%$ CI 1.2, 4.0) and women of South-East Asian descent (adjusted OR 1.9; 95\% CI 1.0, 3.7); Middle Eastern women had the lowest association between LGA and EGWG, with a non-statistically significant adjusted OR of 1.5 (95\% CI $0.9,2.4)$. EGWG accounted for $12.6 \%$ of the predicted variance of the outcome LGA $\left(\chi^{2} 140.3, d f 16\right.$, Nagelkerke's $\left.R^{2}=0.126, p<0.001\right)$ in the logistic regression model.

The unadjusted OR of macrosomia was 2.9 (95\% CI 2.1, 3.9) for women with EGWG compared with those within IOM guidelines; the adjusted OR was 2.3 (95\% CI 1.6, 3.3). Adjustment for postdates delivery ( $>40$ weeks) did not

Table 1 Comparison of baseline characteristics in women who gained below, according to and in excess of IOM GWG targets

\begin{tabular}{|c|c|c|c|c|}
\hline Baseline characteristic & $\begin{array}{l}\text { Less than IOM } \\
(n=918 ; 30.3 \% \text { of } \\
\text { GDM pregnancies) }\end{array}$ & $\begin{array}{l}\text { Consistent with IOM } \\
(n=962 ; 31.7 \% \text { of } \\
\text { GDM pregnancies })\end{array}$ & $\begin{array}{l}\text { In excess of IOM } \\
\text { ( } n=1151 ; 38.0 \% \text { of } \\
\text { GDM pregnancies) }\end{array}$ & $p$ value \\
\hline Age, years & $32.7 \pm 5.2$ & $32.2 \pm 5.1$ & $31.5 \pm 5.6$ & $<0.001$ \\
\hline Gestational age at diagnosis, weeks & $26.3 \pm 6.1$ & $27.2 \pm 5.6$ & $27.4 \pm 5.9$ & $<0.001$ \\
\hline Gravidity & $2.9 \pm 1.8$ & $2.8 \pm 1.8$ & $3.1 \pm 2.1$ & $<0.001$ \\
\hline Parity & $1.3 \pm 1.4$ & $1.3 \pm 1.4$ & $1.5 \pm 1.6$ & $<0.01$ \\
\hline \multicolumn{5}{|c|}{ Ethnicity (\% of IOM category) [\% of ethnic group] } \\
\hline European descent & $173(25.4)$ & $192(28.2)$ & $315(46.3)$ & \\
\hline Middle Eastern & $173(21.3)$ & $211(26.0)$ & $428(52.7)$ & $<0.001$ \\
\hline East and South-East Asian & $434(41.3)$ & $406(38.6)$ & $212(20.2)$ & \\
\hline South Asian & $108(32.1)$ & $107(31.8)$ & $121(36.0)$ & \\
\hline Other & $30(19.9)$ & $46(30.4)$ & $75(49.7)$ & \\
\hline Prepregnancy BMI, kg/m² & $25.1 \pm 6.8$ & $25.0 \pm 5.5$ & $28.4 \pm 6.4$ & $<0.001$ \\
\hline Fasting BGL on antenatal OGTT, mmol/1 & $5.0 \pm 0.8$ & $5.0 \pm 0.7$ & $5.4 \pm 0.9$ & $<0.001$ \\
\hline $2 \mathrm{~h}$ BGL on antenatal OGTT, mmol/1 & $8.9 \pm 1.3$ & $8.8 \pm 1.3$ & $8.6 \pm 1.5$ & $<0.001$ \\
\hline $\mathrm{HbA}_{1 \mathrm{c}}$ at GDM diagnosis & & & & $<0.001$ \\
\hline $\mathrm{HbA}_{1 \mathrm{c}}, \%$ & $5.2 \pm 0.5$ & $5.3 \pm 0.6$ & $5.4 \pm 0.7$ & \\
\hline $\mathrm{HbA}_{1 \mathrm{c}}, \mathrm{mmol} / \mathrm{mol}$ & $33 \pm 5.5$ & $34 \pm 6.6$ & $36 \pm 7.7$ & \\
\hline
\end{tabular}

Data are expressed as mean $\pm \mathrm{SD}$ or $n(\%)$

Statistical significance for continuous values tested using one-way ANOVA with Bonferroni adjustment for multiple comparisons 
Table 2 ORs of adverse pregnancy outcomes in EGWG compared with appropriate GWG

\begin{tabular}{|c|c|c|c|}
\hline Adverse outcome & $\begin{array}{l}\text { Outcome in IOM } \\
\text { EGWG, } n(\%)\end{array}$ & $\begin{array}{l}\text { Unadjusted OR } \\
(95 \% \mathrm{CI})\end{array}$ & $\begin{array}{l}\text { Adjusted OR }{ }^{\mathrm{a}} \\
(95 \% \mathrm{CI})\end{array}$ \\
\hline Need for insulin therapy & 481/1151 (41.8) & $1.7(1.5,2.0)^{* * *}$ & $1.2(0.9,1.6)$ \\
\hline Preterm delivery & $96 / 1151(8.3)$ & $1.1(0.9,1.5)$ & $1.0(0.7,1.1)$ \\
\hline Caesarean section & $335 / 1151(29.1)$ & $1.4(1.2,1.7)^{* * * *}$ & $1.5(1.2,1.9)^{* *}$ \\
\hline Shoulder dystocia & $10 / 1014(1.0)$ & $3.4(1.2,10.0)^{*}$ & $3.0(0.7,12.6)$ \\
\hline LGA & 248/1147 (21.6) & $2.0(1.6,2.5)^{* * *}$ & $1.8(1.4,2.4)^{* * * *}$ \\
\hline Macrosomia & 187/1151 (16.2) & $2.9(2.1,3.9)^{* * *}$ & $2.3(1.6,3.3)^{* * * *}$ \\
\hline SGA & $62 / 1149(5.4)$ & $0.6(0.4,0.8)^{* * *}$ & $0.5(0.3,0.7)^{* * * *}$ \\
\hline Neonatal hypoglycaemia & $85 / 1014(8.4)$ & $1.3(0.9,1.8)$ & $1.2(0.8,1.7)$ \\
\hline Neonatal jaundice & 106/1014 (10.5) & $1.9(0.7,1.2)$ & $1.0(0.7,1.3)$ \\
\hline Abnormal postpartum OGTT & $140 / 599(23.4)$ & $1.3(1.0,1.6)$ & $1.3(0.9,1.8)$ \\
\hline
\end{tabular}

${ }^{\text {a }}$ Adjusted for age, ethnicity, gestational age at diagnosis, prepregnancy BMI, gravidity, parity, fasting BGL, $2 \mathrm{~h}$ $\mathrm{BGL}$ and $\mathrm{HbA}_{1 \mathrm{c}}$

${ }^{*} p<0.05,{ }^{* *} p<0.01,{ }^{* * * *} p<0.001$

significantly alter the adjusted OR for LGA or macrosomia, nor did adjusting for prior GDM regarding insulin use in women exceeding IOM GWG targets. EGWG was associated with a lower risk of SGA (OR 0.6 ; $95 \%$ CI $0.4,0.8)$; the adjusted OR was $0.5(95 \% \mathrm{CI} 0.3,0.7)$. There was no increase in risk of preterm delivery, neonatal hypoglycaemia, neonatal jaundice or abnormal postpartum OGTT as a result of EGWG, compared with women who had appropriate GWG.

A sensitivity analysis was performed excluding women with recurrent GDM pregnancies. Similar outcomes were observed in those exceeding IOM GWG targets compared with all pregnancies (electronic supplementary material [ESM] Table 1).

There was overlap within and between each BMI category in the distribution of GWG among women who had SGA, AGA and LGA infants (Fig. 1). With the exception of

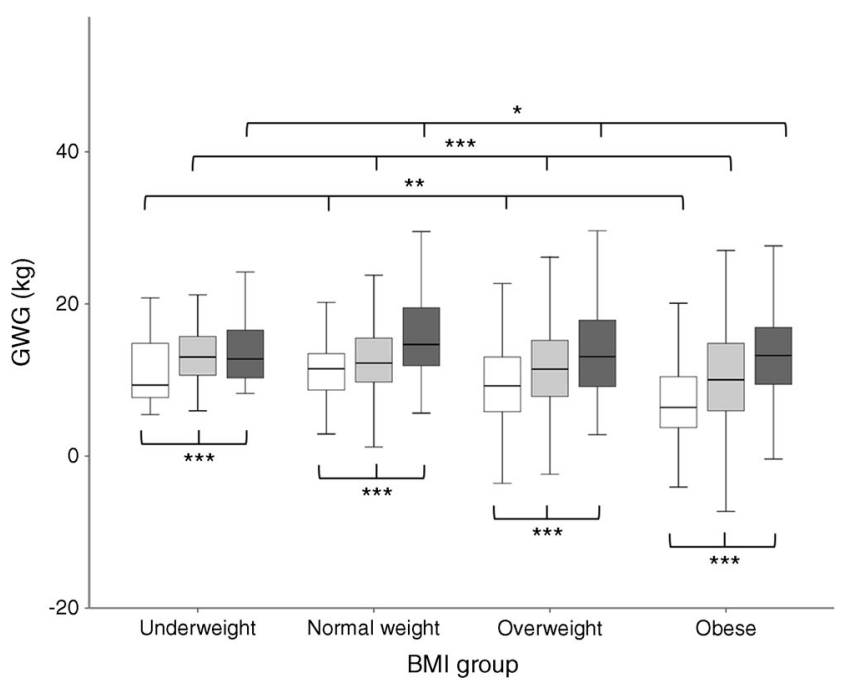

Fig. 1 One-way ANOVA with Bonferroni correction used to compare birthweight categories within each BMI group (significance below box plots) and AGA infants between BMI groups (significance above box plots). White boxes, SGA infants; light grey boxes, AGA infants; dark grey boxes, LGA infants. ${ }^{*} p<0.05,{ }^{* * *} p<0.01,{ }^{* * * *} p<0.001$ underweight women, the mothers of SGA and LGA infants had the lowest and highest median weight gains, respectively, in each BMI category $(p<0.001)$. The odds of LGA significantly increased as GWG increased in overweight and obese women. Overall there was a trend to an increased odds of LGA with BMI and GWG ( $p$ for interaction=0.052).

\section{The effect of modifying weight gain targets on pregnancy outcomes in women who achieved appropriate GWG}

Subtracting $2 \mathrm{~kg}$ from the upper IOM target Subtraction of $2 \mathrm{~kg}$ from the upper IOM limit resulted in a smaller proportion of women achieving appropriate GWG compared with IOM in most BMI categories. Women achieving appropriate GWG with these modified criteria compared with IOM criteria were $23.9 \%$ vs $35.9 \%(p<0.001)$ in healthy weight women, $17.9 \%$ vs $31.4 \%(p<0.001)$ in overweight women and $11.6 \%$ vs $21.6 \%(p<0.001)$ in obese women. There was no statistical difference in underweight women. Compared with IOM targets, modified GWG targets did not achieve any significant differences in the rates of insulin therapy, preterm delivery, shoulder dystocia, Caesarean section, LGA, macrosomia, SGA, neonatal hypoglycaemia, neonatal jaundice or postpartum maternal dysglycaemia (Table 3).

Subtracting $2 \mathrm{~kg}$ from the upper and lower IOM targets Subtraction of $2 \mathrm{~kg}$ from both the upper and lower IOM limits resulted in a greater proportion of women with healthy BMI achieving appropriate GWG compared with IOM targets $(42.6 \%$ vs $35.9 \%, p<0.001)$. By contrast, the proportion of overweight women who achieved this modified GWG target range was significantly lower than the proportion who achieved the IOM targets $(26.7 \%$ vs $31.4 \%, p<0.05)$. Modifying the criteria in this way did not affect the proportion of women gaining appropriate GWG among underweight or 
Table 3 Comparison of maternal and neonatal outcomes according to IOM and modified criteria in women with appropriate GWG.

\begin{tabular}{lllll}
\hline Outcome & IOM & IOM upper limit minus 2 kg & $\begin{array}{l}\text { IOM upper/lower } \\
\text { limits minus 2 kg }\end{array}$ & $\begin{array}{l}\text { Interquartile weight } \\
\text { range for AGA infants }\end{array}$ \\
\hline Insulin use & $288 / 961(30.0)$ & $170 / 591(28.8)$ & $263 / 993(26.5)$ & $484 / 1508(32.1)$ \\
Preterm delivery & $74 / 962(7.7)$ & $47 / 591(8.0)$ & $75 / 993(7.6)$ & $116 / 1508(7.7)$ \\
Caesarean section & $216 / 960(22.5)$ & $130 / 591(22.0)$ & $233 / 993(23.5)$ & $374 / 1508(24.8)$ \\
Shoulder dystocia & $3 / 879(0.3)$ & $2 / 540(0.4)$ & $2 / 901(0.2)$ & $7 / 1363(0.5)$ \\
LGA & $116 / 959(12.1)$ & $75 / 591(12.7)$ & $116 / 993(11.7)$ & $200 / 1505(13.3)$ \\
Macrosomia & $61 / 962(6.3)$ & $40 / 591(6.8)$ & $55 / 993(5.5)$ & $116 / 1508(7.7)$ \\
SGA & $89 / 962(9.3)$ & $63 / 591(10.7)$ & $98 / 991(9.9)$ & $127 / 1507(8.4)$ \\
Neonatal hypoglycaemia & $56 / 879(6.4)$ & $31 / 540(5.7)$ & $52 / 901(5.8)$ & $89 / 1363(6.5)$ \\
Neonatal jaundice & $97 / 879(11.0)$ & $60 / 540(11.1)$ & $105 / 901(11.7)$ & $164 / 1363(12.0)$ \\
Abnormal postpartum OGTT & $166 / 600(27.7)$ & $101 / 385(26.2)$ & $168 / 649(25.9)$ & $241 / 935(25.8)$ \\
\hline
\end{tabular}

Data are expressed as $n(\%)$

$p=\mathrm{ns}$ across all columns

Interquartile ranges were 10.5-15.5 kg (BMI $\left.<18.5 \mathrm{~kg} / \mathrm{m}^{2}\right), 9.5-15.5 \mathrm{~kg}\left(\mathrm{BMI} 18.5-25.0 \mathrm{~kg} / \mathrm{m}^{2}\right), 8.0-15.0 \mathrm{~kg}\left(\mathrm{BMI}>25 \mathrm{~kg} / \mathrm{m}^{2} \mathrm{but}<30 \mathrm{~kg} / \mathrm{m}^{2}\right), 6.0$ $15.0 \mathrm{~kg}\left(\mathrm{BMI} \geq 30.0 \mathrm{~kg} / \mathrm{m}^{2}\right)$

obese women. There were no significant differences in the rates of insulin use, preterm delivery, shoulder dystocia, Caesarean section, LGA, macrosomia, SGA, neonatal hypoglycaemia, neonatal jaundice or postpartum dysglycaemia in the women who achieved the modified criteria of subtracting $2 \mathrm{~kg}$ from the upper and lower limit of IOM compared with IOM targets (Table 3).

Use of the interquartile range (25th-75th percentile) of maternal GWG of women with AGA infants When the interquartile range of maternal GWG of women with AGA infants was applied as a target range to the entire cohort, the following was observed. First, compared with IOM a larger proportion of women achieved appropriate GWG according to this modified criterion in all BMI classes except for the underweight category. Women achieving appropriate GWG with this modified criterion compared with IOM criteria were $52.0 \%$ vs $35.9 \%(p<0.001)$ in healthy weight women, $46.9 . \%$ vs $31.4 \%(p<0.001)$ in overweight women and $49.6 \%$ vs $21.6 \%(p<0.001)$ in obese women. Again, despite more women achieving appropriate GWG, the rates of insulin use, preterm delivery, shoulder dystocia, Caesarean section, LGA, macrosomia, SGA, neonatal hypoglycaemia, neonatal jaundice and postpartum maternal dysglycaemia were not significantly different when compared with IOM targets (Table 3).

ESM Table 2 compares adjusted ORs of excess vs adequate GWG for IOM guidelines and the three modified criteria categories given above.

Use of a 0-4 kg target in class II and III obesity A total of 294 women had class II or III obesity. Only $13.9 \%$ of women gained appropriate GWG according to modified criteria and
$18.7 \%$ with the IOM target of 5-9 kg. The difference was not statistically significant. There were no differences in the rates of insulin use, preterm delivery, shoulder dystocia, Caesarean section, LGA, macrosomia, SGA, neonatal hypoglycaemia, neonatal jaundice or postpartum dysglycaemia in women who gained this recommended amount of weight compared with IOM guidelines (ESM Table 3).

\section{Discussion}

To our knowledge, this is the first study to investigate whether adjustments to IOM targets could improve pregnancy outcomes in women with GDM. Maternal GWG inherently includes a component of fetal and placental weight gain, which is thought to be about $7.5 \mathrm{~kg}$; hence, an appropriate amount of GWG is important for fetal well-being [8]. However, consistent with other studies of pregnancies affected [11] and unaffected by GDM [17], EGWG according to IOM criteria was common, affecting $38.0 \%$ of pregnancies. Additionally, women in the obese category of prepregnancy BMI had an even greater likelihood of EGWG (58.5\% pregnancies), despite lower average GWG, due to the lower allowable GWG in obese women. Interestingly, the mean GWG in the obese cohort $(10.9 \pm 7.5 \mathrm{~kg})$ was already higher than the $9 \mathrm{~kg}$ maximum suggested by the IOM.

Only $31.7 \%$ of patients overall were able to gain weight within IOM targets, consistent with another Australian study which found that only $28 \%$ of women were able to achieve IOM GWG targets [18]. Similarly, that study also found a greater propensity to increased and more variable GWG in obese women compared with women in lower BMI categories. In a prior study at our institution, most GWG occurred 
before the diagnosis of GDM [12]: at GDM diagnosis, onethird of overweight and one-half of obese women had already exceeded the total IOM GWG target intended for the whole of pregnancy. This emphasises the need to focus on healthy GWG targets early in pregnancy, even before the diagnosis of GDM and especially in overweight and obese women. However, studies that have modified individual participants' GWG are not universally associated with improved pregnancy outcomes. A systematic review and meta-analysis of studies on antenatal and lifestyle intervention in obese women that combined antenatal lifestyle, dietary and activity interventions restricted GWG, but there was no robust evidence to show an improvement in the rate of Caesarean section or macrosomia [19]. Further studies since 2012 also show a modest or no effect on limiting GWG and varying effects on neonatal outcomes. The Limiting Weight Gain in Overweight and Obese Women during Pregnancy to Improve Health Outcomes (LIMIT) randomised controlled trial found that antenatal lifestyle advice in overweight and obese women did not reduce the mean GWG or the risk of LGA infants [20]. The Vitamin D and Lifestyle Intervention for GDM Prevention (DALI) study [21] showed antenatal healthy eating intervention was associated with lower GWG and fasting BGL compared with physical activity alone. The recent Finnish Prevention of GDM through Lifestyle Modification (RADIEL) study showed that lifestyle intervention was associated with a lower incidence of GDM and with a $-0.58 \mathrm{~kg}$ reduction in GWG $(95 \% \mathrm{CI}+1.12,-0.04 \mathrm{~kg} ; p=0.037)$ [22].

Following adjustment, EGWG above IOM recommendations conferred an almost twofold increase in the risk of LGA and macrosomia, and a 1.5-fold increase in Caesarean section. There was no association between EGWG and other pregnancy outcomes or postpartum OGTT result. EGWG reduced the risk of having an SGA infant; however, the rate of SGA in women who gained weight according to IOM (9.3\% risk) was similar to the population risk of SGA (10\%). There was significant variation in the risk of EGWG and resultant LGA according to ethnicity. East and South-East Asian women were least likely to exceed IOM GWG, although they had a similar risk of LGA compared with European women. Middle Eastern women had the highest rate of EGWG and yet the lowest association with LGA. Furthermore South Asian women had the highest risk of LGA (2.5-fold risk) when exceeding IOM GWG recommendations, although a lower risk of actually exceeding the GWG targets. Our study findings are consistent with other observational studies, including the Atlantic Diabetes in Pregnancy (Atlantic-DIP) study [11], in which EGWG resulted in an adjusted OR of 2.01 (95\% CI 1.2, 3.3) for LGA.

We evaluated the effect of adjusting the IOM GWG targets. Either a lower or a similar proportion of women were able to achieve acceptable GWG by subtracting $2 \mathrm{~kg}$ from the upper limit or by subtracting $2 \mathrm{~kg}$ from both upper and lower limits of the IOM criteria. This was not surprising, as some of these modified criteria applied tighter ranges for appropriate GWG. When using the interquartile weight range for AGA infants, which by definition spans two quartiles of weight distribution, about $50 \%$ of women were able to achieve 'appropriate' GWG. Obese women were less likely to achieve appropriate GWG compared with healthy weight women according to either IOM or any modified criteria.

The rates of adverse pregnancy outcomes were comparable in women who achieved appropriate GWG when IOM was evaluated against the four modified criteria we assessed. We hypothesise a number of possibilities as to why modification to the IOM GWG ranges, including a more restrictive range, did not lead to significant lowering of adverse outcomes for those who gained weight within these modified ranges. First, only a relatively small percentage of women succeeded in gaining weight according to IOM guidelines; hence, an even smaller proportion of women fell into the generally more restrictive target ranges. Conceivably, if more women had been able to achieve GWG targets for IOM (possibly with intervention commencing earlier in pregnancy), the modified targets would have then led to lower rates of adverse outcomes. Second, there was considerable overlap between GWG among women who had LGA, AGA and SGA infants in each BMI category. Only $12.6 \%$ of the variance of LGA could be predicted by GWG alone. While GWG is an important independent predictor of adverse pregnancy outcome, most notably LGA, a number of other factors have been associated, including maternal glycaemia, particularly in the late third trimester, history of LGA, maternal BMI [23] and maternal hypertriglyceridaemia [24]. Finally, this study reported a $12.2 \%$ rate of LGA among women who gained weight according to IOM guidelines, which is only slightly above the background rate in pregnancies not complicated by GDM. Any improvement in LGA rate would thus be small and a much larger cohort would be required to provide adequate power to detect a difference in outcomes. A total of 7682 cases would be required to detect a $2 \%$ reduction in incidence of LGA (ClinCalc.com. Sample size calculator. Available from http://clincalc.com/stats/samplesize.aspx, accessed 24 July 2016) .

A limitation of this study was the lack of glycaemic control data on women following the diagnosis of GDM, as this might have affected both maternal and fetal outcomes. Another limitation is that potential errors of self-reported weight might have altered the magnitude of GWG. Strengths include a large sample size spanning two decades of data collection, with consistent diagnostic criteria, treatment targets and an endocrinologist supervising the GDM clinic.

In conclusion, our data do not support a recommendation to alter IOM GWG guidelines for women with GDM. Nonetheless, more restrictive targets may still be considered, especially among obese women, to minimise postpartum weight retention and thus long-term maternal morbidity 
related to obesity and type 2 diabetes in this population. Finally, as a large proportion of women exceed the targets set by IOM, further focus is needed on lifestyle interventions that are effective in achieving the current IOM GWG targets in pregnancies with and without GDM. However, it is evident that any measure to reduce the impact of GWG needs to be commenced early in pregnancy. As obesity itself may lead to adverse perinatal outcomes, the role of weight loss prior to conception also needs to be explored further.

Acknowledgements We wish to thank all of the diabetes educators who have collected data and maintained the database over the 22 years of data collection: N. Edghill, C. Finneran, C. Fung, M. Griffiths, G. Holters, M. Horne, J. Mackenzie, R. Myszka, A. O'Neill, A. Oni, J. Payne, M. Stephens, C. van Geloven, J. Warrender and A. Watt, all from the Diabetes Centre, Bankstown-Lidcombe Hospital, Bankstown, NSW, Australia. We would also like to thank J. Gardosi, West Midlands Perinatal Institute, Birmingham, UK, for use of the customised percentile chart calculator.

Data availability The data that support the findings of this study are available from the corresponding author upon reasonable request.

Funding This research received no specific grant from any funding agency in the public, commercial or not-for-profit sectors.

Duality of interest The authors declare that there is no duality of interest associated with this manuscript.

Contribution statement TW was responsible for study conception and design, background research, interpretation of the data, writing and editing the manuscript. RAB was responsible for conception and design, drafting and critical revision of the manuscript. GPR was involved in conception and design, supervision, drafting and critical revision of the manuscript. NWC was responsible for conception and design, supervision, interpretation of the data, drafting and critical revision of the manuscript. JRF was responsible for conception and design, background research, acquisition of data, administrative, technical and material support, supervision, drafting and critical revision of the manuscript. TW is the guarantor of this work. All authors have approved the final version of this manuscript.

\section{References}

1. Ferrara A (2007) Increasing prevalence of gestational diabetes: a public health perspective. Diabetes Care 30(Suppl 2):S141-S146

2. Centre for Epidemiology and Evidence (2014) New South Wales mothers and babies 2012. Available from www.health.nsw.gov. au/hsnsw/Pages/mothers-and-babies-2012.aspx. Accessed 24 July 2016

3. National Research Council, Committee on Maternal Nutrition, Food and Nutrition Board. (1970) Maternal nutrition and the course of pregnancy. Washington, DC: National Academy of Sciences

4. Institute of Medicine (1990) Nutrition during pregnancy. Part 1: weight gain. Part II: nutrient supplements. National Academy Press, Washington, DC
5. Institute of Medicine (2009) Weight gain during pregnancy: reexamining the guidelines. The National Academies Press, Washington, DC

6. DeVader S, Neeley H, Myles T, Leet T (2007) Evaluation of gestational weight gain guidelines for women with normal prepregnancy body mass index. Obstet Gynecol 110:745-751

7. Crozier SR, Inskip HM, Godfrey KM et al (2010) Weight gain in pregnancy and childhood body composition: findings from the Southampton Women's Survey. Am J Clin Nutr 91:1745-1751

8. Kiel DW, Dodson E, Artal R, Boehmer TK, Leet TL (2007) Gestational weight gain and pregnancy outcomes in obese women: how much is enough? Obstet Gynecol 110:752-758

9. Atral R, Lockwood CJ, Brown HL (2010) Weight gain recommendations in pregnancy and the obesity epidemic. Obstet Gynecol 115:152-155

10. Scholl T, Hediger M, Schall JI, Ances I, Smith WK (1995) Gestational weight gain, pregnancy outcome and postpartum weight retention. Obstet Gynecol 86:423-427

11. Egan AM, Dennedy MC, Al-Ramli W, Heerey A, Avalos G, Dunne F (2014) ATLANTIC-DIP: excessive gestational weight gain and pregnancy outcomes in women with gestational or pregestational diabetes mellitus. J Clin Endocrinol Metab 99:212-219

12. Barnes RA, Edghill N, Mackenzie J et al (2013) Predictors of large and small for gestational age birthweight in offspring of women with gestational diabetes mellitus. Diabet Med 30:1040-1046

13. Flack JR, Ross GP, Ho S, McElduff A (2010) Recommended changes to diagnostic criteria for gestational diabetes: impact on workload. Aust N Z J Obstet Gynaecol 50:439-443

14. National Diabetes Service Scheme/Diabetes Australia. Australian diabetes map. Available from www.ndss.com.au/diabetes-map. Accessed 7 Nov 2015

15. Hoffman L, Nolan C, Wilson JD, Oats JJN, Simmons D (1998) Gestational diabetes mellitus - management guidelines. The Australasian Diabetes in Pregnancy Society. Med J Aust 169:93-97

16. Martin FI (1991) The diagnosis of gestational diabetes. Med J Aust 155:112

17. Chu SY, Callaghan WM, Bish CL, D'Angelo D (2009) Gestational weight gain by body mass index among US women delivering live births, 2004-2005: fueling future obesity. Am J Obstet Gynecol 200:271.e1-e7

18. Wong VW, Russell H (2013) Weight gain during pregnancy in women with gestational diabetes: how little is too little? Diabetes Res Clin Pract 102:e32-e34

19. Oteng-Ntim E, Varma R, Crocker H, Poston L, Doyle P (2012) Lifestyle interventions for overweight and obese pregnant women to improve pregnancy outcomes: systematic review and metaanalysis. BMC Med 10:10-17

20. Dodd JM, Turnbull D, McPhee AJ et al (2014) Antenatal lifestyle advice for women who are overweight or obese: LIMIT randomised trial. BMJ 348:g1285

21. Simmons D, Jelsma JG, Galjaard S et al (2015) Results from a European multicenter randomized trial of physical activity and/or healthy eating to reduce the risk of gestational diabetes mellitus: the DALI lifestyle pilot. Diabetes Care 38:1650-1656

22. Koivusalo SB, Rönö K, Klemetti MM et al (2016) Gestational diabetes mellitus can be prevented by lifestyle intervention: the Finnish Gestational Diabetes Prevention Study (RADIEL). Diabetes Care 39:24-30

23. Schaefer-Graf UM, Kjos SL, Kilavuz O et al (2003) Determinants of fetal growth at different periods of pregnancies complicated by gestational diabetes mellitus or impaired glucose tolerance. Diabetes Care 26:193-198

24. Son GH, Kwon JY, Kim YH, Park YW (2010) Maternal serum triglycerides as predictive factors for large-for-gestational age newborns in women with gestational diabetes mellitus. Acta Obstet Et Gynecol Scand 89:700-704 